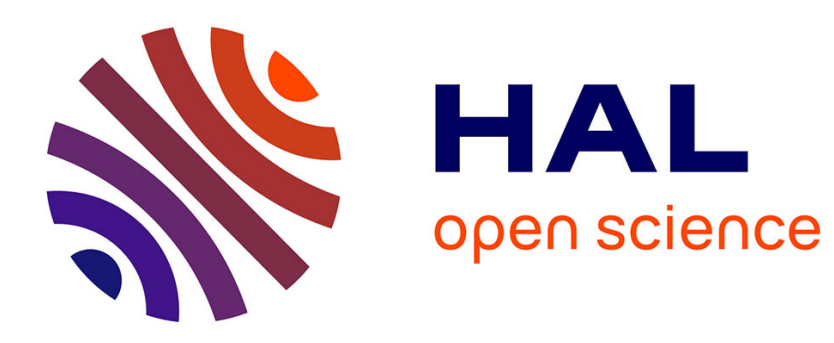

\title{
Profils des entreprises en matière de RSE et innovation technologique
}

Rachel Bocquet, Caroline Danièle Mothe

\section{To cite this version:}

Rachel Bocquet, Caroline Danièle Mothe. Profils des entreprises en matière de RSE et innovation technologique. Revue management \& avenir, 2013, 8 (66), pp.132-151. 10.3917/mav.066.0132 . hal00950166

\section{HAL Id: hal-00950166 \\ https://hal.science/hal-00950166}

Submitted on 25 Feb 2014

HAL is a multi-disciplinary open access archive for the deposit and dissemination of scientific research documents, whether they are published or not. The documents may come from teaching and research institutions in France or abroad, or from public or private research centers.
L'archive ouverte pluridisciplinaire HAL, est destinée au dépôt et à la diffusion de documents scientifiques de niveau recherche, publiés ou non, émanant des établissements d'enseignement et de recherche français ou étrangers, des laboratoires publics ou privés. 


\section{Profils des entreprises en matière de RSE et innovation technologique}

\section{Résumé}

Cet article explore la nature de la relation entre la Responsabilité Sociale des Entreprises (RSE) et l'innovation technologique, avec pour objectif d'identifier les éventuelles spécificités des Petites et Moyennes Entreprises (PME). En dépit de son importance, cette relation reste peu étudiée et les travaux actuels ne permettent pas de différencier les entreprises au regard de la variété des pratiques de RSE mises en œuvre. Afin de contribuer à combler ce vide, nous élaborons un cadre théorique dérivé de la littérature en management stratégique qui distingue deux profils d'adoption des entreprises en matière de RSE. Nous illustrons notre cadre conceptuel par une recherche qualitative auprès de sept PME et filiales de grandes entreprises en Rhône-Alpes. Les résultats montrent que l'innovation technologique des entreprises dépend moins de la taille que de leur comportement stratégique ou réactif en matière de RSE.

Mots clés : Innovation, PME, Responsabilité Sociale des Entreprises (RSE) 


\title{
Firm's profiles in terms of CSR and technological innovation
}

\begin{abstract}
This article explores the nature of the relationship between Corporate Social Responsibility (CSR) and technological innovation, with the objective of identifying possible specificities of Small and Medium Enterprises (SMEs). Despite its importance, this relationship remains poorly studied and current work does not differentiate between firms with regard to the variety of their CSR practices. As a contribution to filling this gap, we develop a theoretical framework derived from the literature in strategic management, which distinguishes between two profiles of CSR adoption. We illustrate our framework with a qualitative research on seven SMEs and subsidiaries of large firms in the Rhone-Alpes region. Results show that firms' technological innovation depends less on the size than on their behavior (strategic or reactive) in terms of CSR.
\end{abstract}

Keywords : Corporate Social Responsibility (CSR), Innovation, SME’s 


\section{Introduction}

La Responsabilité Sociale des Entreprises (RSE) fait l'objet d'une attention croissante dans la littérature depuis une décennie. Cette littérature a mis l'accent sur les différentes dimensions (et critères de mesure) de la RSE, sur les déterminants de son adoption, sur les spécificités de la RSE dans les PME, et sur le lien entre RSE et performance de l'entreprise.

Notre objecettif est d'explorer la relation entre RSE et innovation (Midttun, 2006) en nous concentrant sur l'identification des spécificités potentielles des PME. Les recherches en management stratégique ont montré la relation potentiellement forte entre innovation et RSE ou entre RSE et Développement Durable ${ }^{1}$ (DD) (McWilliams et Siegel, 2000, Moore et Spence, 2006; Husted et Allen, 2007a; Maxfield, 2008 ; Berger-Douce, 2011 ; Ingham et al., 2011). Très récemment, Berger-Douce (2011) montre, à partir d'une étude de cas, que le DD est un vecteur clé de l'innovation des PME et qu'un management responsable et les convictions affirmées du dirigeant permettent de concilier des innovations d'exploitation et d'exploration (ou une « ambidextrie au niveau de l'innovation », Brion et al., 2008 ; Mothe et Brion, 2008). Cette recherche permet d'avancer que le profil stratégique du dirigeant a une influence sur l'innovation réalisée par la PME. Dans la même veine, Mathieu et Soparnot (2007) identifient, au niveau théorique, que le processus d'appropriation du DD peut être vu comme un phénomène intégratif pouvant générer des comportements socialement responsables générateurs d'innovation. Toutefois, cette relation doit encore être affinée dans la mesure où les travaux antérieurs, tant théoriques qu'empiriques, ont rarement appréhendé ce lien sous l'angle de la variété des pratiques de RSE mises en œuvre par les entreprises. En effet, on peut supposer que, dans la lignée des travaux de Miles et Snow (1978) pour caractériser le comportement stratégique des entreprises ou expliquer leurs différences de performance, cette relation entre RSE et innovation peut différer selon leur profil d'adoption en matière de RSE.

Nous tentons de combler partiellement ce vide en élaborant un cadre d'analyse qui distingue deux profils d'entreprises (réactif versus stratégique) au regard des pratiques de RSE mises en œuvre. Nous illustrons notre cadre conceptuel par une étude qualitative exploratoire, qui s'appuie sur sept études d'entreprises de tailles différentes afin d'identifier les spécificités potentielles des PME. Nous discutons ces résultats avant de conclure et d'émettre des suggestions pour des recherches futures.

\section{La littérature sur la RSE en management stratégique}

"Les entreprises intègrent les préoccupations sociales et environnementales à leurs activités commerciales et leurs relations avec les parties prenantes sur une base volontaire " (Commission des Communautés européennes, 2001, p. 6). Cette définition, communément acceptée dans la littérature, insiste sur le caractère volontaire de la RSE. Elle peut être étendue pour caractériser l'innovation responsable par cette intégration volontaire de considérations sociales et environnementales dans la mise au point, la production et la commercialisation d'un produit, service, procédé, système ou mode d'organisation (Ingham et al., 2011). Différents facteurs favorisent l'adoption de pratiques de RSE, notamment le dirigeant et son profil stratégique dans les petites entreprises (e.g., Paradas, 2005 ; Berger-Douce, 2007), la pression des clients (Quairel et Auberger, 2005) ou l'appartenance à un réseau type groupement de PME (Bonneveux et Saulquin, 2009). Nous cherchons ici, non pas à étudier

\footnotetext{
${ }^{1}$ Berger-Douce (2011) rappelle la grande proximité des concepts de RSE et de DD dans le champ du management stratégique où la RSE est définie comme la traduction managériale du DD, en tant que forme d'investissement stratégique (Mc Williams et al., 2006).
} 
les déterminants de l'adoption de pratiques responsables, mais l'impact d'une telle adoption sur l'activité d'innovation des entreprises.

Des travaux récents en management stratégique ont cherché à comprendre en quoi de telles pratiques, au-delà des contraintes légales (McWilliams et Siegel, 2001), peuvent être favorables à l'activité d'innovation des entreprises. S'engager dans des programmes sociaux, sociétaux ou environnementaux semble pouvoir fournir de précieuses ressources (Sharma et Vredenburg, 1998) et favoriser l'innovation produit (Moss Kanter, 1999; Post et al., 2000; Husted, 2005). L'implication des employés jouerait aussi un rôle clé pour la stratégie environnementale, parce qu'elle accroît l'innovation de procédé (Sharma et Vredenburg, 1998). Si la RSE peut être considérée comme un moteur « clé de l'innovation » (Nidumolu et al., 2009, p. 57), les travaux en management stratégique restent hétérogènes et sont encore loin de constituer un paradigme dominant (Lockett et al., 2006). D’un côté, la littérature s'est penchée sur le lien entre une composante de la RSE, à savoir les pratiques environnementales, et l'innovation technologique. De l'autre, des recherches ont testé l'influence de typologies de comportements stratégiques sur la performance des organisations. Toutefois, les recherches à la croisée de ces deux courants, reliant donc le comportement stratégique des entreprises en matière de RSE à la performance en termes d'innovation technologique sont inexistantes. Si deux études ont analysé l'impact de la RSE sur l'innovation (Wagner, 2010 ; Gallego-Alvarez et al., 2011), elles n’introduisent pas le caractère plus ou moins stratégique de l'adoption desdites pratiques de RSE. 
Aussi, la grande majorité des travaux sur le concept de RSE reste de nature conceptuelle (voir par exemple : Jenkins, 2006; Moore et al., 2009; Murillo et Lozano, 2006; Tencati et al., 2004; etc.) car, comme expliqué par Wagner (2010), la RSE est un concept beaucoup plus large que le management environnemental, ce qui rend l'utilisation de mesures plus larges plus complexe.

Dans la terminologie de Porter et Kramer (2006), deux profils d'adoption de RSE peuvent être distingués. D’un côté, un profil d'adoption de type réactif en tant que réponse de l'entreprise à des contraintes ou à des pressions de la part de ses parties prenantes. De l'autre, la RSE comme un véritable levier stratégique. Selon ces auteurs, le profil d'adoption stratégique va au-delà de la mise en œuvre de bonnes pratiques (et du respect de la législation, notamment en matière sociale). La RSE stratégique requiert un alignement entre les pratiques de RSE et la stratégie des entreprises, créant ainsi un cercle vertueux permettant aux activités d'innovation de se développer. Burke et Logsdon (1996) ont transposé l'approche stratégique en termes de RSE, affirmant que l'engagement dans des actions sociales, sociétales et/ou environnementales permet de créer des opportunités de création de valeur. Le cadre d'analyse élaboré permet d'établir un lien fort entre le profil stratégique en matière de RSE et l'innovation technologique. En effet, comme souligné par Husted et Allen (2007, p. 597), "la création de valeur est nécessairement reliée à l'innovation”. La création de valeur apparaît non seulement lorsque les consommateurs et clients sont disposés à payer plus cher des produits ou services en raison de leur nature responsable (innovation en produits) mais aussi lorsque les ressources sont combinées de manière différente et nouvelle, permettant ainsi d'accroître leur efficience (innovation en procédés). La relation entre la RSE et l'innovation technologique serait donc bien établie: la RSE stratégique est un levier clé de l’innovation en produits et/ou en procédés.

Burke et Logsdon (1996) ont défini cinq dimensions stratégiques pour caractériser plus précisément ce lien. Selon eux, la création de valeur, à travers ce modèle, requiert un alignement positif de l'ensemble de ces dimensions :

- centralité: la proximité et l'alignement des pratiques de RSE avec les missions et objectifs de l'entreprise;

- proactivité: le degré d’anticipation des pratiques de RSE par rapport aux tendances sociales émergentes;

- volontarisme: le degré de prise de décision discrétionnaire en matière de RSE et l'absence de contraintes externes imposées;

- visibilité: des pratiques de RSE observables et reconnaissables par l'ensemble des parties prenantes externes et internes;

- appropriation: la capacité à capturer des bénéfices privés associés à la RSE par l'entreprise.

Ces cinq dimensions sont utilisées pour différencier les entreprises qui ont des pratiques RSE " stratégique », c'est-à-dire des pratiques alignées (avec un score élevé sur l'ensemble des dimensions) de celles ayant une RSE « réactive ».

Le modèle de Burke et Logsdon (1996) a pour la première fois été testé par Husted et Allen (2007), dont l'étude empirique sur 110 grandes entreprises espagnoles démontre que seules trois variables stratégiques en matière de RSE (visibilité, appropriation, et volontarisme) sont importantes pour l'innovation (la centralité et la proactivité ne jouant pas). Toutefois, leur étude est limitée aux grandes entreprises et ignore les stratégies en matière de RSE par les PME. Dans la littérature sur les PME, ces dernières sont souvent appréhendées comme ayant des caractéristiques spécifiques du fait de leur taille, notamment par leurs pratiques beaucoup plus informelles que les grandes entreprises, ce qui laisserait supposer que leurs pratiques en matière de RSE peuvent aussi différer (Jenkins, 2006). 
Notre étude empirique cherche donc à explorer d'une part, les spécificités éventuelles des petites entreprises en matière de comportement et de déploiement de la RSE et d'autre part, l'impact potentiel de différents types de comportements stratégiques en matière de RSE sur l'innovation technologique (soit, au sens de Ingham et al., 2011, d'innovation technologique responsable). En effet, Ingham et al. (2011) concluent que les PME rencontrent parfois des difficultés particulières tout en disposant d'atouts leur permettant de saisir des opportunités spécifiques, notamment pour servir des niches. Les auteurs insistent notamment sur le fait que les PME peuvent développer des innovations responsables «à condition toutefois d'en faire le cœur de leur engagement concurrentiel » (ibid, p. 397). Berger-Douce (2007) identifie quatre types de comportements stratégiques en matière d'engagement environnemental et social (appelé « engagement sociétal ») des entrepreneurs, distinguant les stratégies réactives des stratégies proactives en matière environnementale. Dans la lignée de ces travaux, nous cherchons à préciser l'aspect stratégique lié à ces pratiques d'innovation technologique responsable.

\section{Sept études de cas sur les liens entre RSE et innovation}

Si la relation entre RSE et innovation a été testée (Wagner, 2010 ; Gallego-Alvarez et al., 2011), c'est essentiellement l'impact de la RSE sur l'innovation qui a fait l'objet de recherches empiriques. Dans sa recherche doctorale, Ferauge (2011) analyse également les conditions dans lesquelles l'innovation peut favoriser et promouvoir la responsabilité sociétale. Quel que soit le sens de la relation étudiée, les recherches quantitatives, encore peu nombreuses, sont soumises à une limite en matière économétrique (McWilliams et Siegel, 2000) liée au fait que la RSE peut être considérée, en soi, comme une innovation (Vilanova et al., 2009, Husted et Allen, 2007). Par ailleurs, les mesures utilisées pour caractériser la RSE d'un côté (avec l'utilisation d'index), et l'innovation (essentiellement la R\&D) de l'autre, restent à améliorer. En effet, elles s’avèrent peu adaptées aux PME qui n'ont pas toujours des pratiques de RSE directement observables ni des activités formelles de R\&D pour innover. Pour aller plus loin sur le concept de RSE, encore soumis à de multiples définitions et débats, et en explorer le caractère multiforme, ce sont essentiellement des recherches empiriques qualitatives (en particulier dans le Journal of Business Ethics) qui ont été menées (e.g. Jenkins, 2006; Moore et al., 2009; Murillo et Lozano, 2006; Tencati et al., 2004, etc.). Nous avons également choisi cette méthode, en nous appuyant sur l'analyse de sept entreprises françaises.

\subsection{Méthodologie}

Nous avons mené des études de cas sur sept entreprises situées dans la région Rhône-Alpes en France. Ces entreprises, connues pour leurs comportements exemplaires en matière de RSE, ainsi que pour leurs activités d'innovation, ont été choisies avec l'aide de différents experts (Chambre de Commerce et d'Industrie de la Haute-Savoie, associations industrielles, business angels, réseaux d'innovation, etc.). Nous avons aussi recherché des articles dans la presse professionnelle liés à la RSE abordant des cas exemplaires de stratégies d'entreprise en matière de RSE et d'innovation en Rhône-Alpes. Enfin, l'un des chercheurs est dans une institution qui s'appuie sur un Club d'Entreprises regroupant 60 entreprises représentatives de la région. Le président de cette association a été également mobilisé pour aider à identifier les entreprises. Enfin, nous avons également fait appel à la méthode «boule de neige » (Ter Wal et Boschma, 2009), les premières personnes interrogées nous dirigeant vers d'autres entreprises connues pour leur implication en matière de RSE (cette implication n'apparaissant 
pas toujours à l'extérieur chez les PME, qui parfois ne communiquent pas sur cette dimension).

Les entreprises sont de tailles diverses, ce qui permet d'identifier un éventuel effet de la taille sur les pratiques en matière de RSE. Quatre entreprises sont des PME (selon la définition européenne, soit moins de 250 salariés), une cinquième est une filiale de taille moyenne d'un groupe international (Pilot), la sixième une grande filiale d'un groupe mondial (Salomon), et le dernier un groupe de taille moyenne de 5400 salariés (Somfy). Pour les études de cas, nous nous sommes appuyés sur les articles de presse, les rapports internes, les sites Internet et avons triangulé ces données au moyen de 15 entretiens, d'une durée d'environ 2 heures chacune (cf. tableau 1). Ces entretiens, menés en septembre et octobre 2009, se sont appuyés sur la grille d'entretien qui figure en Annexe 1. Ils avaient pour objectif d'une part de cerner la spécificité des comportements en matière de RSE des entreprises de petite taille, d'autre part d'explorer l'éventuel lien entre le profil d'adoption de comportements de RSE (stratégique versus réactif) et l'innovation technologique de l'entreprise. Tous les entretiens ont été enregistrés et ont fait l'objet d'une retranscription exhaustive. L'exploitation des données primaires ainsi collectées a été basée sur une analyse de contenu complétée par l'extraction de verbatims. De manière plus précise, pour déterminer le comportement plus ou moins stratégique en matière de RSE, nous avons réalisé un codage manuel thématique selon les 5 dimensions du modèle de Burke et Logsdon (1996), à savoir centralité, proactivité, volontarisme, visibilité et spécificité. Nous avons d'autre part caractérisé l'activité d'innovation (intensité, type) et évalué, grâce aux verbatims, le lien potentiel entre RSE et innovation. Par ailleurs, nous avons effectué une comparaison entre les entretiens menés auprès de PME et ceux menés auprès de grandes entreprises afin d'identifier des spécificités en fonction de la taille.

Tableau 1: Liste et nature des entretiens des 7 études de cas

\begin{tabular}{|c|c|c|c|}
\hline $\begin{array}{c}\text { Raison sociale de } \\
\text { l'entreprise }\end{array}$ & \begin{tabular}{|c|} 
Activité et taille \\
(nombre d'employés)
\end{tabular} & $\begin{array}{c}\text { Nombre et nature des } \\
\text { entretiens }\end{array}$ & $\begin{array}{c}\text { Statut de } \\
\text { l'entreprise }\end{array}$ \\
\hline Sunea & $\begin{array}{l}\text { Paneaux solaires, } 5 \\
\text { personnes }\end{array}$ & 2 (les fondateurs) & Indépendante \\
\hline Trialp & $\begin{array}{l}\text { Tri et collecte de } \\
\text { déchets, } 120 \text { employés }\end{array}$ & $\begin{array}{l}2 \text { (Président Directeur } \\
\text { Général et Directeur } \\
\text { Général) }\end{array}$ & Indépendante \\
\hline Inddigo & $\begin{array}{l}\text { Ingéniérie durable, } 140 \\
\text { employés }\end{array}$ & 2 (Vice-Présidents) & Indépendante \\
\hline Routin & $\begin{array}{l}\text { Boissons, } 150 \\
\text { employés }\end{array}$ & $\begin{array}{l}1 \text { (Président Directeur } \\
\text { Général) }\end{array}$ & Indépendante \\
\hline $\begin{array}{l}\text { Pilot Corporation of } \\
\text { Europe }\end{array}$ & $\begin{array}{l}\text { Filiale du groupe Pilot } \\
\text { (2400 employés), } \\
\text { solutions d’écriture, } \\
185 \text { employés }\end{array}$ & $\begin{array}{l}2 \text { (secrétaire général, } \\
\text { contrôleur en charge de } \\
\text { la RSE) }\end{array}$ & $\begin{array}{l}\text { Filiale d'un } \\
\text { groupe japonais }\end{array}$ \\
\hline Salomon S.A. & $\begin{array}{l}\text { Filiale d'Amer Sports } \\
\text { (6400 employés), } \\
\text { sports de montagne, } \\
950 \text { employés }\end{array}$ & $\begin{array}{l}4 \text { (Directeur des } \\
\text { Ressources Humaines, } \\
\text { directeur des services } \\
\text { généraux et du Comité } \\
\text { de la Santé et de la } \\
\text { Sécurité au Travail, }\end{array}$ & $\begin{array}{l}\text { Filiale d'un } \\
\text { groupe finlandais }\end{array}$ \\
\hline
\end{tabular}




\begin{tabular}{|l|l|l|l|}
\hline & & $\begin{array}{l}\text { assistante au Directeur } \\
\text { des Ressources } \\
\text { Humaines) }\end{array}$ & \\
\hline Somfy S.A. & $\begin{array}{l}5400 \text { employés, } \\
\text { automatismes pour } \\
\text { maisons et immeubles } \\
\text { France et Vice-Président } \\
\text { communication) }\end{array}$ & $\begin{array}{l}\text { Groupe français } \\
\text { (maison-mère) }\end{array}$ \\
\hline 7 études de cas & $\begin{array}{l}\text { 4 PME, 1 petite filiale, } \\
\text { 1 grand filiale et un } \\
\text { groupe de taille } \\
\text { moyenne }\end{array}$ & 15 entretiens & \\
\hline
\end{tabular}

\subsection{Résultats}

Outre la perception de la RSE par les entreprises, les résultats sont liés à la présence et à l'importance des cinq dimensions de Burke et Logsdon (1996). En effet, comme souligné par Bertoin Antal et Sobczak (2007), il n'y a aucune raison de considérer, a priori, qu'il existe une spécificité française, même si Husted et Allen (2007) ont constaté que seules trois des cinq dimensions s'inscrivent dans l'approche de la RSE des entreprises espagnoles.

\subsubsection{Définition de la RSE}

Afin de bien comprendre ce que signifiait la RSE pour les différentes entreprises interrogées, nous leur avons tout d'abord demandé une définition spontanée de la RSE. Puis, nous avons fourni aux répondants une définition plus fine de la RSE reposant sur les cinq dimensions du modèle pour qu'ils puissent situer leurs pratiques au regard de ces dernières. Un premier constat est celui du manque de consensus sur la terminologie RSE, les répondants utilisant des mots aussi variés tels que "pratiques responsables ou sociales " (Sunea), "responsabilité sociétale » (Routin) ou encore «développement durable » (Somfy). Les petites entreprises insistent davantage sur leur implication dans l'environnement local (territoire), tandis que les grandes ont une vision plus large de leur environnement - «la planète » (Somfy) ou « la société tout entière » (Routin). En revanche, toutes les entreprises s'accordent sur le caractère volontaire de la RSE, au-delà des exigences strictement légales. Le périmètre de la RSE dépend largement de la taille de l'entreprise. Les petites entreprises sont plus enclines à se concentrer sur les parties prenantes internes et sur l'aspect social de la RSE. Les grandes entreprises tendent à avoir une approche plus large et plus économique, surtout orientée vers les acteurs qui permettront d'accroître le rendement économique (comme les clients et les fournisseurs).

\subsubsection{Profil d'adoption stratégique ou réactif}

Pour qualifier le profil d'adoption (réactif ou stratégique) des entreprises en matière de RSE, nous étudions leur position vis-à-vis des quatre premières dimensions (centralité, proactivité, volontarisme, visibilité). La dernière dimension, la spécificité, nous permet ensuite d'établir le lien avec l'innovation des entreprises. Le tableau 2 propose une synthèse des résultats commentés ci-après. 
Tableau 2 : Les 5 dimensions de la RSE dans les 7 entreprises

\begin{tabular}{|l|c|c|c|c|c|}
\hline \multicolumn{1}{|c|}{ Entreprise } & $\begin{array}{c}\text { Centralité } \\
\text { Proximité à la } \\
\text { mission de } \\
\text { l'entreprise }\end{array}$ & $\begin{array}{c}\text { Spécificité } \\
\text { Capacité à } \\
\text { capturer des } \\
\text { bénéfices } \\
\text { privés }\end{array}$ & $\begin{array}{c}\text { Proactivité } \\
\text { Degré de } \\
\text { planification }\end{array}$ & $\begin{array}{c}\text { Volontarisme } \\
\text { Prise de } \\
\text { décision } \\
\text { discrétionnaire }\end{array}$ & $\begin{array}{c}\text { Visibilité } \\
\text { Observable } \\
\text { par les parties } \\
\text { prenantes }\end{array}$ \\
\hline Sunea & +++ & ++ & +++ & ++ & ++ \\
\hline Trialp & +++ & ++ & +++ & +++ & ++ \\
\hline Inddigo & +++ & +++ & +++ & +++ & +++ \\
\hline Routin & + & + & + & + & + \\
\hline $\begin{array}{l}\text { Pilot Corporation } \\
\text { of Europe }\end{array}$ & +++ & +++ & +++ & ++ & ++ \\
\hline Salomon S.A. & + & + & + & + & - \\
\hline Somfy S.A. & ++ & ++ & ++ & ++ & ++ \\
\hline
\end{tabular}

Source: Adapté de Burke et Logsdon, 1996

\section{Centralité}

A la question de savoir si la RSE est alignée sur les missions et objectifs stratégiques de l'entreprise, les dirigeants interrogés (y compris des très petites entreprises, et de celles qui, comme Sunea, ont été créées avec un objectif social, celui de l'intégration des personnes handicapées, ou Trialp, qui est une entreprise d'insertion) ont déclaré que l'objectif premier est d'être rentable, même si «autre chose» (aspects moraux et éthiques) est nécessaire:

"L'objectif est d'être rentable dans un environnement concurrentiel rude. L'objectif est également de proposer un nouvel emploi aux personnes handicapées qui sont limités dans leur choix d'activité » (Sunea).

«L'objectif de l'entreprise est de générer des euros, mais il ne faut pas que cela! L'emploi est très important mais, ce qui est essentiel, c'est de donner du sens au travail! »(Trialp).

Cet objectif de rentabilité (à court terme), ne saurait à lui seul fournir une bonne mesure de l'alignement sans la reconnaissance du rôle clé joué par la direction générale :

"Le principal levier est la direction générale, par son effet d'entraînement de l'équipe. Nous devons faire en sorte que nos collaborateurs se développent et leur fournir les conditions afin qu'ils deviennent autonomes et responsables » (Trialp).

De l'avis des répondants, l'intégration de la RSE aux missions et objectifs stratégiques de l'entreprise comporte deux obstacles principaux :

"Les principaux obstacles sont de deux types: il y a un besoin de temps, qui nécessite de la volonté, une vision stratégique et du sens donné à l'action. Et la RSE requiert des processus transversaux et une personne qui a la légitimité pour gérer l'ensemble du système (donc qui se trouve au niveau de la direction générale »(Inddigo).

\section{Proactivité}

Les entreprises proactives cherchent à anticiper sur les tendances de l'environnement pour développer un avantage concurrentiel à long terme:

«La RSE est une base pour des performances à long terme: investir aujourd'hui pour de meilleurs rendements économiques dans l'avenir. Elle nous aide à rester en tête de la compétition » (Trialp). 
«La RSE est un levier stratégique pour développer de nouvelles affaires, techniques, modèles sociaux, locaux de gestion des territoires (plutôt que des objectifs quantitatifs pour la croissance). Nous voulons être innovateurs avec une capacité d'influence » (Indiggo).

"Par exemple, agir au niveau local dans l'enseignement donne des opportunités à nos employés de se sentir fiers de leur action. Les propriétaires [de l'entreprise] ont toujours été dans cette démarche » (Routin).

\section{Volontarisme}

Certaines entreprises ne parviennent pas à être dans une démarche volontariste car elles réagissent aux contraintes externes et aux incitations de l'environnement:

"Si la RSE était, avant, un levier stratégique unique ; elle est désormais au cour de l'activité d'écriture car elle est imposée par les clients (Migros, par exemple)»(Pilot).

"La RSE est une réponse aux troubles sociaux suite à la restructuration et à diverses acquisitions, ce qui a réduit le personnel de 1.600 à 950 salariés: la RSE offre une nouvelle vision »(Salomon).

\section{Visibilité}

De nombreuses entreprises ont adopté des normes formelles telles que celles de l'ISO 9001, 14000 ou SD 21000 (Afnor), afin de «faire ce que vous dites » (Somfy). Les répondants s'accordent pour reconnaître l'importance de la codification des codes de conduite car elle suppose l'engagement de tous. La plupart des personnes interrogées soulignent également la nécessité de disposer de critères objectifs pour mesurer l'engagement de l'entreprise dans la RSE et suivre les processus, non seulement en raison du temps et de l'argent nécessaires (facteur souligné par les PME), mais aussi parce que de nombreuses actions ne sont pas encore quantifiables. Les dirigeants de PME ont d'ailleurs des difficultés à trouver les bons indicateurs tout en insistant sur l'importance «du faire ». De manière générale, la formalisation des pratiques de RSE doit être en rapport avec la taille de l'entreprise et l'activité. Les propos suivants illustrent ces différents points :

"Nous disposons de 3 critères: le nombre de personnes employées, le degré de satisfaction des employés, et la redistribution des bénéfices selon la règles des 3 tiers »(Sunea).

«La question des indicateurs est la plus difficile. Les indicateurs sont une priorité, en termes de propriété du capital, d'insertion, mais je ne peux pas vous donner des indicateurs objectifs réels, nous devons travailler sur cette question » (Trialp).

"Pour nous, les indicateurs ne sont pas essentiels, la clé est de faire. Mais les clients les demandent de plus en plus » (Routin).

"Dans ce processus d'amélioration continue, les indicateurs sont nécessaires, sinon c'est de l'auto-proclamation. Un processus sans indicateurs n'a pas de sens, et les indicateurs sans processus non plus. Un système de gestion formelle est la seule approche possible pour l'amélioration continue » (Inddigo).

«La RSE est intégrée dans nos valeurs, mais pas dans les indicateurs. JSOX ${ }^{2}$ a permis une formalisation importante en termes de gestion des risques, de prévention et de contrôle. L'objectif n'est pas de maîtriser, mais de sécuriser nos processus, ce qui est essentiel pour le respect de notre engagement en matière de RSE »(Pilot).

\footnotetext{
${ }^{2}$ La loi Sarbanes-Oxley (SOX) a été adaptée à la culture japonaise du groupe PILOT, d'où l'acronyme $\mathrm{J}(\mathrm{Japan}) \mathrm{SOX}$ au sein du groupe.
} 
Nous avons également étudié les sites Internet des 7 entreprises interrogées pour obtenir des informations complémentaires sur leurs stratégies de communication en matière de RSE. La plupart des petites entreprises (Sunea, Trialp, Inddigo) donnent à voir leur engagement en matière de RSE, directement sur la page d'accueil. Inddigo met en avant une liste de critères RSE dans son rapport annuel, accessible en ligne. Cependant, ces trois entreprises sont actives dans des secteurs où les dimensions sociales et sociétales sont au cœur de leurs activités. Par conséquent, cet effort de formalisation peut être largement expliqué par l'effet sectoriel.

Les résultats relatifs aux quatre premières dimensions (centralité, proactivité, volontarisme, visibilité) semblent indiquer que la taille des entreprises n'intervient en rien dans leur profil d'adoption en matière de RSE puisque, dans notre échantillon, ce sont surtout des PME (Trialp, Indiggo) qui adoptent des comportements stratégiques en termes de RSE, et ce bien qu'elles adoptent généralement des pratiques plus informelles.

Pour aller plus loin dans notre compréhension du profil d'adoption des entreprises, les répondants ont été invités à identifier les principaux obstacles auxquels ils sont confrontés pour mettre en œuvre leurs pratiques de RSE. La plupart des obstacles sont internes, notamment pour les PME, qui manquent souvent de temps, de ressources humaines et financières (mais ces contraintes financières sont mentionnées par les grandes entreprises aussi, particulièrement en temps de crise). Le profil d'adoption de la RSE semble avoir une influence sur la perception de ces obstacles. Les entreprises dites «stratégiques " (comme Trialp) cherchent à surmonter ces obstacles, les autres, dans une position plus réactive, semblent parfois un peu baisser les bras (Routin) :

"Les obstacles internes sont les plus importants: nous devons sortir de l'agglomération, lancer les gens dans l'autonomie, avoir une culture d'entreprise pour créer un dynamisme, des compétences solides. Nous avons besoin d'évolutions organisationnelles: cela a un coût mais elles sont nécessaires! » (Trialp).

«Le coût des certifications est important: 7 jours par an. Nous n'avons pas adopté la norme ISO 14001 parce que c'est un coût supplémentaire. Nous faisons ce que nous pouvons, en fonction des ressources disponibles. Et nous avons des contraintes importantes en temps de crise » (Routin).

Ce sont les entreprises les plus engagées dans la RSE stratégique qui perçoivent le mieux les obstacles, en insistant d'ailleurs davantage sur ces obstacles que sur les motivations. Aussi, tout comme l'indique la littérature sur les obstacles à l'innovation (voir par exemple Mairesse et Mohnen, 2010), les entreprises confrontées à ces difficultés d'adoption sont les mieux à même d'en évaluer les difficultés de mise en œuvre.

\subsubsection{RSE et innovation (spécificité)}

Les entreprises développent deux lectures de la relation entre la RSE et l'innovation. Pour certaines (les plus innovantes), la RSE est clairement orientée vers l'innovation technologique et la possibilité de contribuer à l'avantage concurrentiel. Le plus souvent, les entreprises tentent de faire les choses différemment d'une manière qui serve au mieux les clients.

"L'idée de créer une chaîne courte pour le traitement des huiles usagées est venue en 20012002 alors que personne ne s'intéressait à ce sujet. Elle a été lancée pour offrir une solution aux clients (stations de sports d'hiver) à des fins de différenciation (marketing). Elle nous a servi en termes de réputation et d'image pour capter de nouveaux clients » (Trialp). 
«La RSE est une source d'avantage concurrentiel. Par rapport à la concurrence, on est en avance sur la RSE. Nous vendons des stylos recyclés au même prix. Ceci constitue une véritable réponse aux besoins du marché. En choisissant de vendre des produits au même prix, nous ne visons pas un retour immédiat, mais avons plutôt une approche à long terme. Ce choix est conforme à nos valeurs. Le rôle du leader est important pour communiquer et transférer ces valeurs, qui sont rarement codifiées »(Pilot).

D'autres entreprises conçoivent la RSE comme un engagement social qui facilite leur apprentissage et leur adaptation. L'accent est mis sur la " compétence sociale » (Ghemawat, 2001) qui peut améliorer les innovations en procédés et, dans une moindre mesure en produits. Cependant, l'effet semble plus limité dans la mesure où les innovations sont surtout incrémentales.

«La RSE permet d'impliquer plus de gens, de les motiver sur le projet d'entreprise. Ainsi, la RSE implique la motivation qui implique l'innovation. Mais l'innovation dans les PME est diffuse, les choses ne peuvent pas facilement être découpées. La volonté d'innovation ne se limite pas à quelques personnes, elle doit être dans toute l'organisation »(Inddigo).

«La RSE permet d'innover en éliminant les craintes, en laissant les gens s'exprimer pour créer de nouvelles règles, de nouvelles façons de faire. Nous pouvons faire mieux à l'aide de notre personnel, et nous devons faire preuve d'innovation car c'est notre façon unique de survivre à l'encontre de nos concurrents »(Routin).

En ce qui concerne la relation entre la RSE et l'innovation, elle semble être dans le sens RSE vers Innovation pour la plupart des entreprises analysées, même si la relation Innovation vers RSE n'est pas à exclure. Cette dernière s'applique davantage aux grandes entreprises, qui ont moins de contraintes financières et pour lesquelles l'innovation peut avoir un effet de levier, et favoriser d'autres innovations, notamment de nature organisationnelle telles que les pratiques de RSE (voir les exemples de la Prius de Toyota et du Crédit Agricole donnés par Porter et Kramer, 2006). Nos résultats, et l'analyse des sites Internet des sept entreprises, nous permettent d'évaluer les cinq dimensions de la RSE (Burke et Logsdon, 1996) dans les sept entreprises ( $c f$. tableau 2):

Étonnamment, dans notre échantillon, les petites entreprises semblent avoir une orientation plus stratégique de la RSE que les grandes entreprises. Le volontarisme par exemple, qui est négativement corrélé à la création de valeur pour les grandes entreprises dans l'étude de Husted et Allen (2007), apparaît ici comme une incitation à l'innovation pour répondre aux exigences de conformité imposées de l'extérieur et créer une valeur partagée (Porter et Kramer, 2011) 


\section{Discussion des résultats}

Dans la plupart des cas observés, les entreprises qui ont un profil stratégique d'adoption en matière de RSE perçoivent cette dernière comme un moyen de différenciation (Siegel et Vitaliano, 2007). Sur la base de notre catégorisation de la RSE, ce profil d'adoption n'est pas celui uniquement des grandes entreprises qui «transforment les activités de la chaîne de valeur au profit de l'entreprise tout en renforçant la stratégie » (Porter et Kramer, 2006, p. 89). En effet, la PME Inddigo fait exception à cela compte tenu du modèle d'affaire et de l'activité même de cette entreprise (ingénierie dans le développement durable).

Deux résultats principaux ressortent de notre étude, résultats qui devraient être approfondis dans le cadre d'études quantitatives sur un large échantillon d'entreprises:

- Le premier résultat a trait à l'objectif central de notre recherche, qui est le lien entre les profils d'adoption de la RSE et l'innovation. La plupart des petites entreprises de notre échantillon semblent utiliser la RSE comme une stratégie sociale qui leur permet de contribuer à améliorer leur performance d'innovation (RSE $\rightarrow$ Innovation). Les PME ayant adopté un profil stratégique en matière de RSE sembleraient en mesure de développer des innovations de produits plus radicales que celles ayant un profil réactif, pour lesquelles les innovations sont le plus souvent de nature incrémentale. Les entreprises ayant un profil stratégique auraient tendance à adopter une approche multipartite large alors que les entreprises réactives en matière de RSE se concentreraient sur les parties prenantes locales et internes comme les employés et les clients (Murillo et Lozano, 2006). Pour ces dernières, comme le suggèrent Porter et Kramer (2006), l'impact semble plus limité dans la mesure où elles n'utilisent pas toutes les opportunités offertes par leur engagement dans les aspects sociaux ou environnementaux, qui pourraient contribuer à «ouvrir » leur processus d'innovation et s'appuyer davantage sur leurs parties prenantes pour innover. Ces entreprises « réactives » répondraient ainsi davantage à un modèle d'innovation fermé sur les parties prenantes internes (essentiellement les salariés) ;

- Le deuxième résultat est lié à l'effet sectoriel, qui semble important pour les petites entreprises. Comme le montre Spence (1999), ce facteur influe sur la culture en matière de RSE. Dans notre cas, trois entreprises (Inddigo, Sunea, Trialp) opèrent toutes dans des secteurs où les compétences sociales et environnementales sont des déterminants clés de l'avantage concurrentiel. Pour ces PME, il est plus facile de s'orienter vers la reconnaissance d'opportunités en matière de RSE dans la mesure où la relation est plus directe et presque évidente. La dimension de centralité, liée à la RSE stratégique, caractérise ces petites entreprises qui suivent les mêmes trajectoires d'évolution que celles empruntées généralement par de grandes entreprises en raison de leur activité - et de leur profil stratégique. Ce résultat dans notre échantillon de petites entreprises devrait ainsi être réexaminé par des recherches futures sur les PME dans d'autres secteurs.

\section{Conclusion}

Cette recherche contribue à un thème peu exploré qui présente des enjeux forts compte tenu de l'intérêt croissant porté à la RSE d'une part, et aux stratégies d'innovation, d'autre part, comme des leviers clés à la croissance des PME. Elle complète utilement les recherches entamées par Ingham et al. (2011) sur les spécificités des PME en matière de développement d'innovations responsables en se focalisant sur le lien entre les profils stratégiques des entreprises en matière de RSE et le type d'innovation technologique.

D'un point de vue théorique, cette recherche s’inscrit dans le champ du management stratégique qui s'écarte d'une perspective purement éthique ou morale pour s'intéresser aux 
comportements stratégiques des entreprises en matière de RSE. Dans notre échantillon, l'innovation des entreprises dépend moins de la taille de l'entreprise, que de son profil (plus ou moins stratégique) d'adoption des pratiques de RSE. Des recherches complémentaires devraient aller plus loin dans les facteurs contextuels, afin de relier la taille, mais également le profil de l'entreprise (et de son dirigeant) aux pratiques de RSE. A notre connaissance, il n'existe pas encore de travaux empiriques qui ont développé une telle perspective. Si l'étude de Bertoin Antal et Sobczak (2007) permet d'identifier les facteurs socio-économiques, culturels et juridiques qui affectent le développement et la forme prise par la RSE en France, leur analyse du cadre institutionnel ne s’appuie sur aucune donnée empirique.

D’un point de vue managérial, nos résultats peuvent conduire les entreprises à mieux identifier leur profil d'adoption des pratiques de RSE et d'évaluer la distance qui leur reste à parcourir au regard des cinq dimensions développées par Burke et Logsdon (1996). Notre recherche qualitative rend compte de l'importance d'un alignement de l'ensemble de ces dimensions pour favoriser l'innovation. Si les entreprises interrogées se sentent concernées par chacune d'elles, rares sont celles qui combinent complètement les cinq et parviennent à un alignement stratégique global, hormis Indiggo (ce qui peut s'expliquer par le métier même de l'entreprise, tourné autour de l'ingénierie en matière de développement durable). Des recherches complémentaires actuellement en cours sur un échantillon large d'entreprises tendent à confirmer empiriquement le lien entre le comportement en matière de RSE et l'innovation technologique, montrant que les entreprises ayant un profil de RSE stratégique innovent plus que les autres, que ce soit en produits ou en procédés.

Les efforts portés à l'élaboration de nouveaux cadres théoriques et modèles empiriques pour préciser le lien entre RSE et innovation (ainsi que son aspect récursif), en lien avec le comportement stratégique des entreprises doivent être poursuivis. Dans cette optique, la prise en compte d'autres dimensions de l'innovation (marketing, organisationnelle, etc.) et de la RSE (social, sociétal ou environnemental) pourrait s'avérer particulièrement fructueuse. Des recherches complémentaires devraient aussi être menées, de manière longitudinale, pour éclairer les conditions du passage du profil d'adoption de la RSE " réactif » au profil "stratégique », tout en les élargissant, à l'instar de Ingham et al. (2011) aux innovations responsables de nature non technologique. D'autres prolongements pourraient être proposés, dans la lignée des travaux conceptuels de Temri (2011) sur les innovations technologiques environnementales dans les petites entreprises, pour analyser les liens entre pratiques de RSE et l'innovation en matière environnementale.

\section{Bibliographie}

Baron D. (2001), « Private Politics, Corporate Social Responsibility and Integrated Strategy », Journal of Economics \& Management Strategy, Vol. 10, p. 7-45.

Berger-Douce S. (2007), « Les stratégies d'engagement sociétal des entrepreneurs », Revue de l'Entrepreneuriat, Vol. 6(1), p. 53-71.

Berger-Douce S. (2011), « Le développement durable, un levier d'innovation pour les PME?», Revue Française de Gestion, Vol. 6(215), p. 147-166.

Berthoin Antal A., Sobczak A. (2007), "Corporate Social Responsibility in France », Business and Society, Vol. 46(1), p. 9-32.

Bonneveux E., Saulquin J-Y. (2009), « L'appropriation de la RSE par les dirigeants de PME. Le réseau comme vecteur de l'apprentissage managérial », Management \& Avenir, Vol. 3(23), p. 170-186. 
Brion S., Favre-Bonté V., Mothe C. (2008), «Quelles formes d'ambidextrie pour combiner innovations d'exploitation et d'exploration ? », Management International, Vol. 12(3), p. 29-44.

Burke L., Logsdon M. (1996), "How Corporate Social Responsibility Pays Off », Long Range Planning, Vol. 29(4), p. 495-502.

Commission of the European Communities (2001), Green Paper «Promoting a European Framework for Corporate Social Responsibility », COM 366 final, Brussels.

Donaldson T., Preston L.E. (1995), «The stakeholder theory of the corporation: Concepts, Evidence, and Implications », Academy of Management Review, Vol. 20(1), p. 65-91.

Ferauge P. (2011), « Responsabilité sociétale du dirigeant et innovation : approche de leur complémentarité dans un contexte de PME», thèse de doctorat, U. de Mons Warocquié, Belgique.

Gallego-Alvarez I., Prado-Lorenzo J.M., Garcia-Sanchez I-M. (2011), « Corporate social responsibility and innovation: A resource-based theory », Management Decision, Vol. 49(10), p. 1709-1727.

Ghoshal S. (2005), «Bad Management Theories Are Destroying Good Management Practices », Academy of Management Learning and Education, Vol. 4(1), p. 75-91.

Granovetter M. (1985), «Economic Action and Social Structure: the Problem of Embeddedness », American Journal of Sociology, Vol. 91(3), p. 481-510.

Hamel G., Prahalad C.K. (1989), "Strategic Intent », Harvard Business Review, p. 63-76, May June.

Hart S.L. (1995), «A Natural-Resource-Based View of the Firm », Academy of Management Review, Vol. 20(4), p. 986-1014.

Husted B.W., Allen D.B. (2007a), «Corporate Social Strategy in Multinational Enterprises: Antecedents and Value Creation », Journal of Business Ethics, Vol. 74, p. 34-361.

Husted B.W., Allen D.B. (2007b), "Strategic Corporate Social Responsibility and Value Creation among Large Firms ", Long Range Planning, Vol. 40, p. 594-610.

Ingham, M., Depret H., M., Hamdouch, A. (2011), L'innovation responsable comme opportunité stratégique: problématisation et illustration dans le cas de PME de la domotique, dans «PME, dynamiques entrepreneuriales et innovation », A. Hamdouch, S. Reboud et C. Tanguy (eds), P.I.E Peter Lang, Collection "Business \& Innovation", 412 p. $\mathrm{n}^{\circ} 1$, ch. 15, p. 379-402.

Jenkins H. (2004), "A Critique of Conventional CSR Theory: An SME Perspective ", Journal of General Management, Vol. 29(4), p. 37-57.

Jenkins H. (2006), " Small Business Champions for Corporate Social Responsibility », Journal of Business Ethics, Vol. 67, p. 241-256.

Jones T.M., Wicks A.C. (1999), « Convergent stakeholder theory, Academy of Management Review », Vol. 24(2), p. 206-221.

Lindgreen A., Swaen V., Maon F. (2009), «Introduction: Corporate Social Responsibility Implementation », Journal of Business Ethics, Vol. 85, p. 251-256.

Lockett A., Moon J., Wayne V. (2006), "Corporate social responsibility in management Research: focus, nature, salience and sources of influence », Journal of Management Studies, Vol. 43, p. 115-36.

Mairesse J., Mohnen P. (2010), « Using Innovations Surveys for Econometric Analysis », Working Paper 15857, Cambridge, Mass: National Bureau of Economic Research.

McWilliams A., Siegel D.S. (2000), « Corporate social responsibility and financial performance: correlation or misspecification? », Strategic Management Journal, Vol. 21(5), p. 603-609. 
McWilliams A., Siegel D.S., Wright P.M. (2006), « Guest Editors’ Introduction: Corporate Social Responsibility: Strategic Implications », Journal of Management Studies, Vol. 43(1), p. 1-18.

Mathieu A., Soparnot R. (2007), «L’appropriation du concept de développement durable en entreprise : un générateur d'innovation », XVIème Conférence Internationale de l’Association Internationale de Management Stratégique, Montréal, 6-9 Juin.

Maxfield S. (2008), « Reconciling Corporate Citizenship and Competitive Strategy: Insights from Economic Theory », Journal of Business Ethics, Vol. 80, p. 367-377.

Midttun A. (2006), « C(S)R and Innovation, Compatibility or Contradiction? » Towards a Dynamic Reinterpretation of C(S)R, Fondazione Eni Enrico Mattei, Paper n³. 2006, December, http://www.feem.it/Feem/Pub/Publications/CSRPapers/default.htm.

Miles R. H., Snow C. C. (1978), « Organizational strategy, structure and process », Mc GrawHill, New-York.

Moss Kanter R. (1999), « From spare change to real change: The social sector as beta site for business innovation », Harvard Business Review, p. 122-132, May-June.

Mothe C., Brion S. (2008), "Innovation : exploiter ou explorer ? », Revue Française de Gestion, Vol. 34(187), p. 101-108.

Murillo D., Lozano J.M. (2006), "SMEs and CSR: An Approach to CSR in their Own Words», Journal of Business Ethics, Vol. 67, p. 227-240.

Moore G., Slack R., Gibbon J. (2009), « Criteria for Responsible Business Practicce in SMEs: An Exploratory Case of U.K. Fair Trade Organisations », Journal of Business Ethics, Vol. 89, p. 173-188.

Moore G., Spence L. (2006), « Editorial: Responsibility and Small Business », Journal of Business Ethics, Vol. 7, p. 219-226.

Nidumolu R., C.K. Prahalad C.K., Rangaswami M.R. (2009), «Why sustainability is now the key driver of innovation », Harvard Business Review, p. 56-64, September.

Ortiz Avram D. and Kühne S. (2008), Implementing Responsible Business Behavior from a Strategic Management Perspective: Developing a Framework for Austrian SMEs », Journal of Business Ethics, Vol. 82(2), p. 463-475.

Paradas, A. (2007), " Le dirigeant comme levier de la RSE en TPE. Approche exploratoire basée sur l'utilisation de récits et d'une cartographie cognitive ", Revue Internationale PME, Vol. 20(3-4), p. 43-67.

Perrini F. (2005), " Building a European Portrait of Corporate Social Responsibility Reporting », European Management Journal, Vol. 23(6), p. 611-627.

Porter M.E., Kramer M.R. (2006), « Strategy and Society », Harvard Business Review, p. 7792, December.

Porter M. E., Kramer M. R. (2011), « Creating shared value », Harvard Business Review, Vol. 89(1/2), p. 62-77.

Postel N., Rousseau S. (2008), « RSE et éthique d'entreprise : la nécessité des institutions », M@n@gement, Vol.11(2),p.137-160.

Quairel F., Auberger M-N. (2005), « Management responsable et PME : Une relecture du concept de "responsabilité sociétale de l'entreprise ", La Revue des Sciences de Gestion, Direction et Gestion, n²11-212, p. 111-126.

Siegel D.S. (2009), Green Management Matters Only If It Yields More Green: An Economic/Strategic Perspective ", Academy of Management Perspectives, p. 5-16, August.

Siegel D.S., Vitaliano D. (2007), « An Empirical Analysis of the Strategic Use of Corporate Social Responsibility », Journal of Economics \& Management Strategy, Vol. 16(3), p. 773-792. 
Spence L.J. (2007), « CSR and Small Business in a European Policy Context: The Five 'C' of CSR and Small Business Research », Agenda 2007, Business and Society Review, Vol. 112(4), p. 533-552.

Spence L., Perrini F. (2008), "Global Overview of Ethics and CSR in SMEs - Europe », International Society of Business, Economics, and Ethics Congress.

Spence L., Rutherfoord R. (2003), "Small Business and Empirical Perspectives in Business Ethics: Editorial », Journal of Business Ethics, Vol. 47, p. 1-5.

Temri, L. (2011), « Innovations technologiques environnementales dans les petites entreprises: proposition d'un modèle d'analyse », Innovations, N³4, p. 11-36.

Tencati A., Perrini F., Pogutz S. (2004), «New Tools to Foster Corporate Socially Responsible Behavior », Journal of Business Ethics, Vol. 53, p. 173-190.

Ter Wal, A.L.J., Boschma, R.A. (2009), " Applying social network analysis in economic geography: framing some key analytic issues », The Annals of Regional Science, Vol. 43(3), p. 739-756.

Thompson J.K., Smith H.L. (1991), « Social responsibility and small business: suggestionsfor research », Journal of Small Business Management, p. 30-44, January.

Vilanova M., Lozano J.M., Arenas D. (2009), « Exploring the Nature of the Relationship Between CSR and Competitiveness », Journal of Business Ethics, Vol. 87, p. 57-69.

Wagner, M. (2010), " Corporate Social Performance and Innovation with High Social Benefits: A Quantitative Analysis », Journal of Business Ethics, Vol. 94, p. 581-594. 


\section{Annexe 1}

\section{Guide d'entretien - RSE et Innovation}

Objectif de l'étude exploratoire:

Participer à une meilleure compréhension du lien entre Responsabilité Sociale de l'Entreprise (RSE) et innovation technologique, en particulier du point de vue des PME. D’où 2 objectifs :

- cerner la spécificité du profil d'adoption de la RSE par les PME

- explorer si, selon le profil d'adoption de l'entreprise, l'impact sur l’innovation est différencié.

\section{Méthodologie :}

Etude qualitative auprès de dirigeants d'entreprises sur la base d'entretiens semi-directifs (en particulier de PME)

\begin{tabular}{|c|c|}
\hline $\begin{array}{l}\text { Partie } 1 \text { : Mieux connaître l'entreprise et son } \\
\text { activité. }\end{array}$ & \\
\hline $\begin{array}{l}\text { Partie } 2 \text { : Mieux définir ce que l’on peut entendre } \\
\text { par « Responsabilité Sociale de l’Entreprise } \\
(\text { RSE)» }\end{array}$ & $\begin{array}{l}\text { 1) Que signifie la RSE de votre point de vue ? } \\
\text { 2) Que signifie la RSE pour une PME ? }\end{array}$ \\
\hline $\begin{array}{l}\text { Partie } 3 \text { : Importance d'une stratégie de RSE du } \\
\text { point de vue de l'activité }\end{array}$ & $\begin{array}{l}\text { 3) Quelle importance accordez-vous à une } \\
\text { stratégie de RSE dans votre stratégie? } \\
\text { 4) Quelles sont les caractéristiques de votre RSE ? } \\
\text { (relancer pour avoir des détails sur chacune des } 5 \\
\text { dimensions du modèle et pouvoir caractériser le } \\
\text { comportement stratégique versus réactif) }\end{array}$ \\
\hline $\begin{array}{l}\text { Partie } 4 \text { : Votre opinion sur l'adoption des } \\
\text { stratégies de RSE }\end{array}$ & $\begin{array}{l}\text { 5) Quelles sont les difficultés dans l'adoption } \\
\text { d'une telle stratégie pour votre entreprise? } \\
\text { 6) Quels sont les facteurs qui encouragent une } \\
\text { telle adoption? }\end{array}$ \\
\hline Partie 5 : RSE, innovation et croissance & $\begin{array}{l}\text { 7) Quelle relation voyez-vous entre RSE et sa } \\
\text { performance (relancer pour avoir une définition de } \\
\text { la performance)? } \\
\text { 8) Comment caractériseriez-vous l'activité } \\
\text { d'innovation de votre entreprise? } \\
\text { 8bis) Quels sont les types et intensité } \\
\text { d'innovation? }\end{array}$ \\
\hline Partie 6 : Lien entre RSE et innovation & $\begin{array}{l}\text { 9) Y a-t-il un lien entre votre stratégie de RSE et } \\
\text { celle d'innovation? } \\
\text { 10) Si oui, pouvez-vous l'expliciter davantage ? } \\
\text { 11) Exemples concrets }\end{array}$ \\
\hline Pour conclure & $\begin{array}{l}\text { 12) Comment votre entreprise voit-elle son avenir } \\
\text { en termes de RSE ? Quelles étapes futures? }\end{array}$ \\
\hline
\end{tabular}


Annexe 2

Extrait de la grille de codage pour l’item « spécificité »

\begin{tabular}{|c|c|c|c|c|c|c|c|}
\hline & 产 & 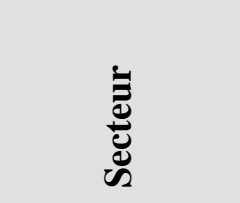 & Verbatim & 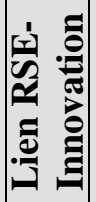 & ơ. & 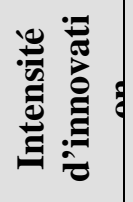 & 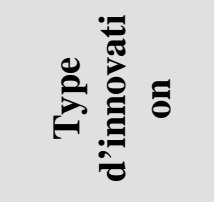 \\
\hline Trialp & PME & $\begin{array}{l}\text { Recyclage, } \\
\text { Traitement des } \\
\text { Déchets }\end{array}$ & $\begin{array}{l}\text { "L'idée de créer une chaîne } \\
\text { courte pour le traitement } \\
\text { des huiles usagées est } \\
\text { venue en 2001-2002 alors } \\
\text { que personne ne } \\
\text { s'intéressait à ce sujet. Elle } \\
\text { a été lancée pour offrir une } \\
\text { solution aux clients } \\
\text { (stations de sports d'hiver) } \\
\text { à des fins de } \\
\text { différenciation a nous a } \\
\text { (marketing). Elle nou } \\
\text { servi en termes de } \\
\text { réputation et d'image pour } \\
\text { capter de nouveaux clients } \\
\text { " }\end{array}$ & +++ & $\begin{array}{l}\text { Différenciation, } \\
\text { image, } \\
\text { réputation, } \\
\text { nouveaux clients }\end{array}$ & +++ & $\begin{array}{l}\text { Inno } \\
\text { technologique } \\
\text { produits }\end{array}$ \\
\hline Indiggo & PME & $\begin{array}{c}\text { Ingéniérie du } \\
\text { développement } \\
\text { durable }\end{array}$ & $\begin{array}{l}\text { "La RSE permet } \\
\text { d'impliquer plus de gens, } \\
\text { de les motiver sur le projet } \\
\text { d'entreprise. Ainsi, la RSE } \\
\text { implique la motivation qui } \\
\text { implique l'innovation. Mais } \\
\text { l'innovation dans les PME } \\
\text { est diffuse, les choses ne } \\
\text { peuvent pas facilement être } \\
\text { découpées. }\end{array}$ & ++ & $\begin{array}{l}\text { Motivation et } \\
\text { implication des } \\
\text { employés }\end{array}$ & $\begin{array}{c}+ \\
\text { (diffus) }\end{array}$ & $\begin{array}{l}\text { Innovation } \\
\text { procédés } \\
\text { surtout }\end{array}$ \\
\hline
\end{tabular}

